\title{
The bank of standardized stimuli (BOSS): comparison between French and English norms
}

\author{
Mathieu B. Brodeur • Eva Kehayia • \\ Geneviève Dion-Lessard • Mélissa Chauret • \\ Tina Montreuil • Emmanuelle Dionne-Dostie • \\ Martin Lepage
}

Published online: 21 February 2012

(C) Psychonomic Society, Inc. 2012

\begin{abstract}
Throughout the last decades, numerous picture data sets have been developed, such as the Snodgrass and Vanderwart (1980) set, and have been normalized for variables such as name and familiarity; however, due to cultural and linguistic differences, norms can vary from one country to another. The effect due specifically to culture has already been demonstrated by comparing samples from different countries where the same language is spoken. On the other hand, it is still not clear how differences between languages may affect
\end{abstract}

Electronic supplementary material The online version of this article (doi:10.3758/s13428-011-0184-7) contains supplementary material, which is available to authorized users.

M. B. Brodeur $\cdot$ T. Montreuil $\cdot$ M. Lepage

Douglas Mental Health University Institute,

Department of Psychiatry, McGill University,

Montréal, Québec, Canada

E. Kehayia

School of Physical and Occupational Therapy,

Jewish Rehabilitation Hospital, McGill University,

Montréal, Québec, Canada

E. Kehayia

Centre for Interdisciplinary Research in Rehabilitation,

Montréal, Québec, Canada

G. Dion-Lessard • E. Dionne-Dostie

Department of Psychology, Université de Montréal,

Montréal, Québec, Canada

M. Chauret $\cdot$ T. Montreuil

Department of Psychology, Université du Québec à Montréal,

Montréal, Québec, Canada

M. B. Brodeur $(\square)$

Douglas Mental Health University Institute, FBC Pavilion,

Department of Psychiatry, McGill University,

6875 Boulevard LaSalle,

Verdun, Québec H4H 1R3, Canada

e-mail: mathieu.brodeur@douglas.mcgill.ca norms. The present study explores this issue by collecting and comparing norms on names and many other features from French Canadian speakers and English Canadian speakers living in Montreal, who thus live in similar cultural environments. Norms were collected for the photos of objects from the Bank of Standardized Stimuli (BOSS) by asking participants to name the objects, to categorize them, and to rate their familiarity, visual complexity, object agreement, viewpoint agreement, and manipulability. Names and ratings from the French speakers are available in Appendix A, available in the supplemental materials. The results show that most of the norms are comparable across linguistic groups and also that the ratings given are correlated across linguistic groups. The only significant group differences were found in viewpoint agreement and visual complexity. Overall, there was good concordance between the norms collected from French and English native speakers living in the same cultural setting.

Keywords Language $\cdot$ Cultural differences - Normative data set Photograph stimuli

Advances in the field of cognitive neuroscience rely predominantly on the quality of the stimuli used in experimental studies. This holds particularly true in studies dedicated to understanding visual, linguistic, and memory processes. For instance, researchers often use normative banks of images in studies that utilize visual agents, to ensure that the stimuli are consistent with those used in analogous studies. The use of normative stimuli is now considered a requisite practice for drawing adequate comparisons across experiments. Snodgrass and Vanderwart (1980) created one of the most influential and widely used sets of visual stimuli in cognitive neuroscience. They published 260 black-andwhite, line-drawn pictures of various concepts, and all of the pictures were normalized by asking participants to name the 
concept and to rate the image for familiarity, visual complexity, and the degree to which the picture matched the participant's mentally generated image of the concept's name.

To validate existing data sets of visual stimuli, researchers around the world have conducted normalization studies in their respective languages. Studies have been carried out in Chinese (Bates et al., 2003; Weekes, Shu, Hao, Liu, \& Tan, 2007; Yoon et al., 2004), French (Alario \& Ferrand, 1999; Bonin, Peereman, Malardier, Méot, \& Chalard, 2003; Schwitter, Boyer, Méot, Bonin, \& Laganaro, 2004), Spanish (Álvarez \& Cuetos, 2007; Bates et al., 2003; Cuetos \& Alija, 2003; Cuetos, Ellis, \& Alvarez, 1999), Japanese (Nishimoto, Miyawaki, Ueda, Une, \& Takahashi, 2005), Portuguese (Miranda, Pompéia, \& Bueno, 2004; Pompéia, Miranda, \& Bueno, 2001, 2003), Italian (Barbarotto, Laiacona, Macchi, \& Capitani, 2002; Bates et al., 2003; Dell'Acqua, Lotto, \& Job, 2000), Dutch (Severens, Van Lommel, Ratinckx, \& Hartsuiker, 2005), Icelandic (Pind, Jónsdóttir, Gissurardóttir, \& Jónsson, 2000; Pind \& Tryggvadóttir, 2002), British English (Barry, Morrison, \& Ellis, 1997; Ellis \& Morrison, 1998), and Bulgarian and Hungarian (Bates et al., 2003).

Linguistic comparisons of norms have been performed across populations from different countries, and thus different cultures. Culture, like language, can have a substantial impact on the recognition and rating of pictures, and these differences occasionally require researchers to adjust their selection of stimuli as a function of the cultural background of their participants. For instance, Alario and Ferrand (1999) excluded objects from the Snodgrass and Vanderwart (1980) set that are uncommon in France, such as the pretzel, the baseball bat, and the American football helmet. These exclusions would not have been necessary when testing Frenchspeaking Canadians, who are frequently exposed to such objects. It can thus be said that culture can generate differences independently of mother tongue. This is well illustrated in studies comparing populations of different origins who speak the same language. Sirois, Kremin, and Cohen (2006) collected normative data for pictures drawn from the standardized European Picture Pool for Oral Naming database with a Canadian French-speaking sample and compared the norms with those collected in France. The findings showed that French Canadian individuals reached a significantly lower mean of name agreement than did French individuals living in France ( $84 \%$ vs. $92 \%)$. Although it was not statistically tested, a similar difference ( $86 \%$ vs. $93 \%$ ) was also seen when comparing the name agreement norms from American (Cycowicz, Friedman, Rothstein, \& Snodgrass, 1997) and British individuals (Barry et al., 1997). Sirois et al. also showed that the mean ratings measured in Canadian French were significantly higher for familiarity (3.31) and lower for visual complexity (2.69) than were the mean ratings collected in France (2.97 and 3.32, respectively). Culture thus appears to be a critical variable that affects norms.
Pompéia et al. (2003) also looked at linguistic differences, but used a used a different analytic approach. They computed the correlations between norms from different countries instead of comparing mean ratings or agreements. The values included in the correlations were the norm values from two different countries, and each point corresponded to one picture. The correlations of name agreement were variable and relatively low, ranging from .185 (between Spain and Japan) to .598 (between Spain and Brazil). On the other hand, the correlations of familiarity ratings were all positive and highly significant, ranging from .711 (between Japan and the U.S.) to .913 (between France and the U.S.). Correlations of visual complexity were also highly significant, ranging from .708 (between Brazil and Spain) to .954 (between France and the U.S.). Therefore, what is familiar and complex in France was found to be mostly familiar and complex in the U.S., highlighting the prototypicality and universality of the concepts depicted in the Snodgrass and Vanderwart (1980) set. This finding is in contrast to the results presented by Sirois et al. (2006) described above, which showed that despite the correlations found across countries, the mean ratings and agreements measured in one country might be qualitatively different from those measured in another country; for instance, a familiar object in France may be even more familiar in the U.S.

The effect of culture has been tested by controlling for language, but to our knowledge, no study has examined how language affects norms while controlling for the cultural setting. One way to address this is by comparing the norms of two different linguistic groups living in the same community, and who are thus exposed to the same objects. Within this context, Viggiano, Vannucci, and Righi (2004) recruited native English speakers and native Italian speakers in colleges and universities in Florence, Italy, to participate in their normative project. However, while the cultural context was the same for all speakers, English speakers had been much less exposed to the Italian culture, since they lived there only transiently and for a short period of time. Thus, it cannot be claimed that these speakers shared the same cultural background as the Italian speakers. Moreover, cross-linguistic comparison was not the main purpose of this study, and groups were not statistically compared. Thus, cross-linguistic effects on norms within a fixed culture are still unknown.

Montreal, Canada, and the particularity of its linguistic environment (French/English) offer a unique opportunity to study the effect of language on normative values within a culturally controlled environment. The present project was designed within this context, to investigate the impact of languages on norms, in an attempt to control for cultural effects. First, norms from French Canadian speakers living in the Montreal area were collected (see Appendix A, provided as supplemental materials). These norms were then 
compared with those collected in a previous study (Brodeur, Dionne-Dostie, Montreuil, \& Lepage, 2010) from English Canadian speakers who also lived in the Montreal area. The normalization procedures were identical in the two studies. Namely, the photos were projected on a screen, and participants were instructed to write the names of the objects, to categorize them, and to rate several features, such as familiarity and visual complexity, on a 5-point rating scale. The English norms can be downloaded along with the Brodeur et al. article. The same 480 high-quality color photos, which compose the Bank of Standardized Stimuli (BOSS), were assessed by each linguistic group. These photos portray common objects in as true-to-life a way as possible. The variables submitted to the normative procedure included the name, familiarity, visual complexity, category, object agreement, viewpoint agreement, and manipulability of the objects.

\section{Method}

\section{Participants}

Two subgroups, including 30 and 28 native French speakers, respectively, were recruited by placing advertisements in local newspapers and online classifieds, such as Craigslist. The participants in the first subgroup included 18 females and 12 males, with a mean age of $30.0( \pm 10.7)$ years and a mean level of education of $14.7( \pm 2.3)$ years. The participants in the second subgroup included 16 females and 12 males, with a mean age of $33.9( \pm 12.0)$ years and a mean level of education of $14.6( \pm 3.5)$ years. The two subgroups underwent normalization using a different set of norms. The first subgroup was tested for name, category, familiarity, and visual complexity, while the second subgroup was tested for object agreement, viewpoint agreement, and manipulability.

\section{Stimuli}

The stimuli included the 480 normative color photos of common objects (e.g., toothbrush, computer mouse, paperclip, etc.) from the BOSS. These objects were all displayed on a white background and set in a frame of 2,000 $\times 2,000$ pixels. The creation procedure for these photos is described in the original article (Brodeur et al., 2010). All objects are listed in Appendix A (see the supplemental materials), and some examples are presented in Fig. 1 (the whole set can be obtained by contacting the corresponding author). The size of the object is also provided in Appendix A, reflected by the percentage of the area covered by the object within the frame.

Normative variables

Name, familiarity, and visual complexity were submitted to normalization. These three variables are systematically included in normative studies, as they are known to have a strong influence on a number of cognitive processes, such as naming (Alario et al., 2004; Barry et al., 1997; Bates et al., 2003; Bonin, Chalard, Méot, \& Fayol, 2002; Cuetos et al., 1999; Rossion \& Pourtois, 2004), categorization (Hannah \& Brooks, 2009), and memory (Begleiter, Porjesz, \& Wang, 1995). Image agreement, the fourth classic normative variable, was not tested, as such. Image agreement refers to the matching between a picture of the set and the image mentally generated after reading the object's name, which to some extent reflects the typicality of the object. Images can be matched with regard to different features, including their design and position (i.e., angle of view). In the present study, participants were asked to rate the matching of these two features separately. In this way, it was anticipated that it would be possible to obtain information about the typicality of the object itself and about the way that it is viewed, which is invaluable information for researchers needing highly recognizable stimuli.

In addition, two new normative variables were added. First, objects had to be classified within predefined categories. Categories characterize objects and are essential for researchers needing specific types of objects. The second variable was the manipulability of the object, which indicates how well objects are associated with specific actions. The nature of the actions is usually undefined; however, in a recent study, van Elk and Blanke (2011) provided this information for 120 objects from the BOSS. Assessing manipulability is indispensable for a set of objects, especially when considering the impact that manipulability can have on reaction times and other cognitive processes, as accounted for in the theory of affordance (Greeno, 1994).

Procedure

The normalization procedure was carried out in a conference room, and the stimuli were presented via an Optoma EP7150 DLP projector $(1,024 \times 768 \mathrm{XGA})$ connected to a

Fig. 1 Sample photos of objects from the Bank of Standardized Stimuli (BOSS)
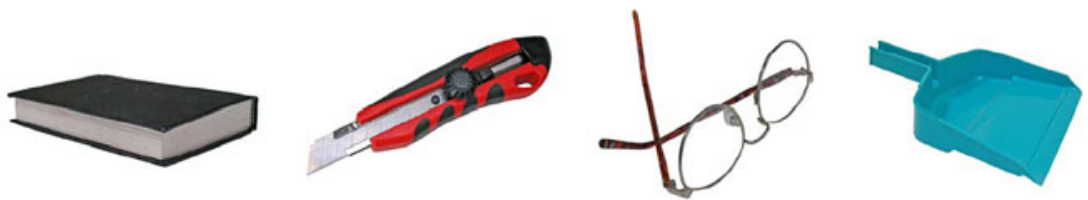
laptop. The projector had a lower resolution than the digital photos, but the quality of the projected images was clear and very satisfactory. The calibration of the RGB color display was verified using the calibratemonspd function of Psychtoolbox (Brainard, 1997) and a SpectraScan-650 colorimeter at the end of the project. The photo presentation was managed with E-Prime software (Psychology Software Tools, Pittsburgh, PA). On the day of testing, up to 12 participants were invited to a conference room. They were handed an instruction sheet and an anonymous response sheet on which they indicated their age, gender, and years of education. Prior to starting the experiment, they received an explanation about the objectives of the study and were provided oral instructions by the experimenter. These instructions are presented in Table 1 in their French and English versions. Note that the instructions were only provided in French for the present study.

Subgroups 1 and 2 received different instructions. The participants of Subgroup 1 were asked to write the name of the object, to indicate its semantic category, and to rate its familiarity and visual complexity. When they were unable to give a name, they were asked to write "CPO," "CPN," or "BDL," depending on whether they (respectively) had no idea what the object was (CPO: ne connaît pas l'objet; i.e., "don't know the object"), knew the object but not its name (CPN: connaît pas le nom; i.e., "don't know the name"), or knew the name but were temporarily unable to retrieve it (BDL: bout de la langue; i.e., "tip of the tongue"). $\mathrm{CPO}, \mathrm{CPN}$, and BDL will be, respectively, referred to by their English equivalents "DKO," "DKN," and "TOT" for the purposes of this publication. Participants also had to classify the objects as belonging to one of 18 categories or to an "other" category. These categories included building materials (matériaux de construction); clothing (vêtements); decorations and gift accessories (accessoires de décoration et cadeaux); electronic devices and accessories (appareils et accessoires électroniques); food (nourriture); furniture (mobilier); games, toys, and entertainment (jeux, jouets et divertissements); hand labor tools and accessories (outils et accessoires de travaux manuels); household articles and cleaners (articles ménagers et produits nettoyants); jewels and money (bijoux et argent); kitchen utensils (accessoires de cuisine); medical instruments and accessories (instruments et accessoires médicaux); musical instruments (instruments de musique); natural elements and vegetation (éléments naturels et végétaux); outdoor activity and sport items (articles de sport et plein air); skin care and bathroom items (articles de toilette et produits de beauté); stationary and school supplies (articles de bureau et articles scolaires); and weapons and items related to war (armes et articles de guerre). It was clearly stated that the "other" category should be used only if none of the proposed categories satisfied the participant's own criteria for the object; the participants were

Table 1 Instructions

Instruction in French

\begin{tabular}{|c|c|}
\hline \multicolumn{2}{|c|}{ Subgroup $1(n=30)$} \\
\hline Name & $\begin{array}{l}\text { Identifiez chaque image de la façon la } \\
\text { plus brève et non ambiguë possible en } \\
\text { écrivant un seul nom, le premier qui } \\
\text { vous vient à l'esprit. }\end{array}$ \\
\hline Category & $\begin{array}{l}\text { Indiquez à quelle catégorie chacun des } \\
\text { objets suivants appartient. }\end{array}$ \\
\hline Familiarity & $\begin{array}{l}\text { Indiquez à quel point vous êtes familier } \\
\text { avec l'objet. }\end{array}$ \\
\hline Visual complexity & $\begin{array}{l}\text { Indiquez à quel point l'image vous } \\
\text { apparaît complexe en ce qui a trait à } \\
\text { la quantité de détails et la complexité } \\
\text { des contours. }\end{array}$ \\
\hline \multicolumn{2}{|c|}{ Subgroup $2(n=28)$} \\
\hline Object agreement & $\begin{array}{l}\text { Indépendamment de sa position, évaluez } \\
\text { le niveau de ressemblance entre l'image } \\
\text { présentée et la représentation mentale } \\
\text { que vous aviez de cet objet. }\end{array}$ \\
\hline $\begin{array}{l}\text { Viewpoint } \\
\text { agreement }\end{array}$ & $\begin{array}{l}\text { Évaluer à quel point l'objet est positionné } \\
\text { tel que vous l'aviez imaginé. }\end{array}$ \\
\hline Manipulability & $\begin{array}{l}\text { Seriez-vous en mesure de mimer l'action } \\
\text { qui est habituellement associée à cet } \\
\text { objet, de manière à ce qu'une personne } \\
\text { qui vous observe faire ce mouvement } \\
\text { soit capable de déterminer l'objet en } \\
\text { question? }\end{array}$ \\
\hline
\end{tabular}

English translation

Identify the object as briefly and unambiguously as possible by writing only one name, the first name that comes to mind.

Determine in which category the object belongs.

Rate the level to which you are familiar with the object.

Subjectively rate the level to which the image appears to be complex in terms of the quantity of details and the intricacy of the lines.

How closely does the picture resemble the mental image you had for the object name, independently from its position?

How closely does the object match the position of the object you imagined?

Could you easily mime the action usually associated with this object so that any person looking at you doing this action could decide which object goes with this action? 
discouraged from using this option if their intention was to be more specific regarding the category. The categories were sorted in alphabetical order on a sheet. Familiarity was rated by circling the proper value on a 5-point rating scale, where 1 meant very unfamiliar and 5 very familiar. Participants were asked to rate the concept itself rather than the picture of the object. Visual complexity was also rated on a 5-point rating scale, with 1 being not complex and 5 being highly complex. For both ratings, participants were asked to use the full range of the scale. They were also told that they were not required to categorize or rate the familiarity and visual complexity of objects that they did not know (instead, they were to respond "DKO," as for the name). Participants provided their response on a sheet after the presentation of each photo. The photos were projected onto a screen for $20 \mathrm{~s}$ each. The pace was established beforehand to allow sufficient time for the participants to write their responses. The order of the stimulus sequence was randomized across groups in order to avoid sequence order effects.

Participants in Subgroup 2 were exposed to a different stimulus presentation. Each trial started with a 5-s presentation of the object's name. The name was the one that had the highest agreement among the participants of Subgroup 1. During the 5-s period, participants had to generate a mental image of the object. The word was followed by a 15-s presentation of the object corresponding to the name. Participants were asked to rate the object agreement - that is, the concordance between the presented object and the object they had imagined, independently from the image's viewpoint. They were also asked to rate the viewpoint agreement by indicating how similar were the positions of the presented and their imagined objects, independently from their resemblance. Responses were provided on a 5point rating scale, with 1 being no concordance and 5 being a high concordance. When the participants were unable to generate a mental image, they were asked to write "PIM," standing for pas d'image mentale (i.e., "no mental image," NMI). Prior to the testing session, two examples were presented to help participants understand the instructions. The word "airplane" was presented, followed by a photo of a Boeing airliner seen from above. In between these two stimuli, another airplane was presented as an example of what a participant could have potentially imagined after reading the word "airplane." In one example, the imagined airplane was a Boeing airliner seen from below. This was presented as a case deserving a high rating for object agreement (i.e., both airplanes were Boeing airliners) but a low rating for viewpoint agreement (i.e., they were seen from different viewpoints). In the second example, the imagined airplane was a Cessna seen from above, which thus deserved a low rating for object agreement (i.e., two different types of airplanes) but a high rating for viewpoint agreement (i.e., they are seen from similar viewpoints). Participants were told that their viewpoint rating should not take into account differences in orientation or reflection between the objects. For instance, the profile of a car pointing toward the right and another profile pointing toward the left are reflected (i.e., mirror) images of each other; however, the angle of view remains the same across these two images, as they display the same amount of information. Accordingly, these two images should deserve a high score on the viewpoint scale. A third variable, manipulability, was also rated on a 5point rating scale. Manipulability was described as the property of an object being associated with a very specific action, which makes this object recognizable. A value of 1 on the scale was assigned to a definite "no" response, and a value of 5 was assigned to a definite "yes" response.

\section{Analyses}

For each object, the frequency of use of the different names was calculated and turned into a percentage. These percentages were calculated after excluding the "DKO," "DKN," and "TOT" responses. The name reaching the highest percentage was the modal name, and its percentage reflected the modal name agreement. The $H$ statistic, reflecting the number and weight of alternative names, is another norm used to measure agreement. It was computed according to the following formula:

$H=\sum_{i=1}^{k} P_{i} \log _{2}\left(1 / P_{i}\right)$,

where $k$ refers to the number of different names given to each picture, and $P_{i}$ refers to the proportion of participants who gave each name for an object. The $H$ statistic of an object with a unique name and no alternatives would be 0 , and the $H$ statistic of an object with two names provided with equivalent frequencies would be 1.00 . This value would be smaller if the alternative name was provided at a lower frequency rate than the modal name. On the other hand, the $H$ statistic would increase as a function of the number of alternatives. For instance, one modal name with $50 \%$ frequency and two alternatives of $25 \%$ frequency each would produce an $H$ value of 1.50 for their object.

Category-related norms were computed following the procedure used for names. A modal category, the category agreement, and an $H_{\text {cat }}$ statistic were thus extracted and will be described in the Results. Norms for the variables rated on a 5-point rating scale were all computed by averaging the ratings and measuring the standard deviations.

Analyses were conducted on objects, not on participants, with the rating or agreement as the dependent variable. Each mean norm value was compared with those from English speakers (data described in Brodeur et al., 2010) by conducting analyses of variance (ANOVAs) with Language (2 
levels) and Category (11 levels) as the between- and withinobject factors, respectively. The Category factor included only categories that included a minimum of 18 objects (see these categories below in Table 3). Including this factor in the ANOVA made it possible to test whether any group difference was driven by a specific category of objects. The ANOVA provides only a partial answer as to the inter-linguistic-group differences, however. As a matter of fact, overall mean ratings for the norms across two linguistic groups can be comparable despite rating differences for individual objects. For example, an object can receive a rating of 5 by the French speakers and a 2 by the English speakers, while another object may be given a rating of 2 by the French speakers and a 5 by the English speakers. Although the ratings are not the same across objects on linguistic groups, their overall mean is the same. To complement the ANOVA and to further test the correspondence between the French- and English-speaker norms, we performed correlational analyses. The correlations were between the rating/ agreement obtained among the French speakers, on the $y$-axis, and the rating/agreement obtained among the English samples, on the $x$-axis. Each point thus corresponded to one of the 480 objects, not to a participant. The alphas for the ANOVAs and the correlations were all Bonferroni corrected, with a corrected alpha threshold of .0038 .

\section{Results}

The mean norms of all objects are summarized in Table 2, along with the standard deviations and the min and max scores. The norms for individual objects are presented in
Appendix A (see the supplemental materials). In this appendix, the photo stimuli are sorted as a function of their English file name, but the French modal names are also included.

Overall, most norms from the French speakers were comparable to those from the English speakers. The percentages of "DKO," "TOT," and "NMI" responses were equivalent across linguistic groups. There were negligible intergroup differences of modal name agreement $(63 \%$ with French speakers vs. 64\% with English speakers), DKN ( $4 \%$ vs. $6 \%)$, category agreement $(76 \%$ vs. $73 \%)$, familiarity (4.1 vs. 4.0$)$, object agreement (4.0 vs. 3.9), and manipulability (2.8 vs. 2.6), but none of these differences was statistically significant. Larger discrepancies were observed between linguistic groups for the $H(1.56$ vs. 1.65$)$ and $H_{\text {cat }}(1.03$ vs. 1.15$)$ values. As previously mentioned, these differences were not statistically significant. The only significant group differences were observed on visual complexity $(2.1$ vs. 2.4$)[F(1,34)=80.1, p<$ $.0001]$ and viewpoint agreement $(4.0$ vs. 3.7) $[F(1,34)=$ $16.8, p=.0002]$.

The participants assigned all objects to a category. Norms as a function of the categories are presented in Table 3. The ANOVAs indicated that Category (those including a minimum of 18 objects) did not interact with the Language factor. On the other hand, each norm was subject to a strong category main effect (all $p \mathrm{~s}<.0001$ ).

Correlations between the French and English norms are reported in Table 2. Most correlations were above .600, and all correlations were highly significant (all $p$ s $<.0001$ ). The manipulability and familiarity ratings reached the highest correlations, and the smallest correlations were for NMI, TOT, and DKO.

Table 2 Summary of results

\begin{tabular}{|c|c|c|c|c|c|c|c|c|c|}
\hline \multirow[t]{2}{*}{ Variables } & \multicolumn{4}{|c|}{ French Speakers } & \multicolumn{4}{|c|}{ English Speakers } & \multirow[t]{2}{*}{ Linguistic Correlations } \\
\hline & Mean & $\mathrm{SD}$ & Min & Max & Mean & $\mathrm{SD}$ & Min & Max & \\
\hline Modal name agreement & $63 \%$ & $24 \%$ & $14 \%$ & $100 \%$ & $64 \%$ & $23 \%$ & $20 \%$ & $100 \%$ & $.618^{* * *}$ \\
\hline$H$ value & 1.56 & 0.97 & 0.00 & 3.83 & 1.65 & 1.01 & 0 & 4.1 & $.690^{* * *}$ \\
\hline DKO & $2 \%$ & $3 \%$ & $0 \%$ & $18 \%$ & $2 \%$ & $3 \%$ & $0 \%$ & $18 \%$ & $.500^{* * *}$ \\
\hline DKN & $4 \%$ & $7 \%$ & $0 \%$ & $48 \%$ & $6 \%$ & $8 \%$ & $0 \%$ & $47 \%$ & $.633^{* * *}$ \\
\hline TOT & $2 \%$ & $4 \%$ & $0 \%$ & $24 \%$ & $2 \%$ & $3 \%$ & $0 \%$ & $18 \%$ & $.303^{* * *}$ \\
\hline Category agreement & $76 \%$ & $19 \%$ & $18 \%$ & $100 \%$ & $73 \%$ & $19 \%$ & $26 \%$ & $100 \%$ & $.770^{* * *}$ \\
\hline$H_{\text {cat }}$ value & 1.03 & 0.66 & 0.00 & 2.84 & 1.15 & 0.65 & 0.00 & 2.70 & $.721^{* * *}$ \\
\hline Familiarity & 4.1 & 0.5 & 2.3 & 5.0 & 4.0 & 0.4 & 3.0 & 4.8 & $.802^{* * *}$ \\
\hline Visual complexity & 2.1 & 0.4 & 1.3 & 4.0 & 2.4 & 0.4 & 1.4 & 4.1 & $.751^{* * * *}$ \\
\hline Object agreement & 4.0 & 0.6 & 2.4 & 4.8 & 3.9 & 0.5 & 2.3 & 4.9 & $.627^{* * * *}$ \\
\hline Viewpoint agreement & 4.0 & 0.5 & 1.8 & 5.0 & 3.7 & 0.5 & 2.2 & 4.8 & $.698^{* * *}$ \\
\hline NMI & $2 \%$ & $4 \%$ & $0 \%$ & $35 \%$ & $2 \%$ & $4 \%$ & $0 \%$ & $30 \%$ & $.215^{* * *}$ \\
\hline Manipulability & 2.8 & 0.8 & 1.2 & 4.8 & 2.6 & 0.8 & 1.2 & 4.5 & $.886^{* * *}$ \\
\hline
\end{tabular}

$\mathrm{DKO}=$ don`t know object; $\mathrm{DKN}=$ don’t know name; TOT $=$ tip of the tongue; NMI $=$ no mental image. ${ }^{* * *} p<.0001$ 
Table 3 Results as a function of category

\begin{tabular}{|c|c|c|c|c|c|c|c|c|c|c|c|c|c|}
\hline Category & $\mathrm{N}$ & NA & $\mathrm{H}$ & DKO & DKN & TOT & $\mathrm{CA}$ & $H_{\text {cat }}$ & Fam & $\mathrm{VC}$ & $\mathrm{OA}$ & VA & Man \\
\hline Building materials & 3 & $61 \%$ & 1.46 & $0 \%$ & $1 \%$ & $0 \%$ & $84 \%$ & 1.25 & 3.5 & 1.8 & 4.2 & 4.1 & 2.0 \\
\hline Clothing & 28 & $71 \%$ & 1.23 & $0 \%$ & $1 \%$ & $0 \%$ & $82 \%$ & 0.81 & 4.4 & 2.0 & 3.9 & 3.9 & 3.2 \\
\hline Decoration and gift accessories & 28 & $58 \%$ & 1.75 & $2 \%$ & $2 \%$ & $1 \%$ & $69 \%$ & 1.36 & 3.9 & 2.4 & 3.7 & 3.8 & 2.4 \\
\hline Electronic devices and accessories & 34 & $55 \%$ & 1.88 & $1 \%$ & $3 \%$ & $1 \%$ & $71 \%$ & 1.32 & 4.2 & 2.3 & 4.1 & 3.8 & 2.8 \\
\hline Food & 78 & $63 \%$ & 1.55 & $2 \%$ & $3 \%$ & $2 \%$ & $79 \%$ & 0.98 & 4.1 & 2.1 & 4.0 & 4.0 & 2.6 \\
\hline Furniture & 3 & $49 \%$ & 2.10 & $2 \%$ & $4 \%$ & $0 \%$ & $39 \%$ & 2.38 & 3.5 & 2.0 & 3.2 & 3.6 & 2.3 \\
\hline Games, toys, and entertainment & 24 & $52 \%$ & 1.99 & $3 \%$ & $5 \%$ & $1 \%$ & $79 \%$ & 0.82 & 3.6 & 2.3 & 3.7 & 3.9 & 2.8 \\
\hline Hand labor tools and accessories & 40 & $59 \%$ & 1.70 & $2 \%$ & $10 \%$ & $4 \%$ & $75 \%$ & 0.93 & 3.8 & 2.1 & 3.9 & 3.8 & 2.9 \\
\hline Household articles and cleaners & 18 & $53 \%$ & 1.96 & $0 \%$ & $4 \%$ & $4 \%$ & $68 \%$ & 1.48 & 4.2 & 1.9 & 4.0 & 4.0 & 3.2 \\
\hline Jewels and money & 9 & $71 \%$ & 1.30 & $2 \%$ & $2 \%$ & $1 \%$ & $67 \%$ & 1.39 & 4.1 & 2.5 & 3.8 & 4.0 & 3.3 \\
\hline Kitchen utensils & 60 & $59 \%$ & 1.79 & $2 \%$ & $7 \%$ & $2 \%$ & $84 \%$ & 0.73 & 4.1 & 2.0 & 4.0 & 4.1 & 3.0 \\
\hline Medical instruments and accessories & 10 & $64 \%$ & 1.66 & $2 \%$ & $4 \%$ & $3 \%$ & $74 \%$ & 1.13 & 3.5 & 2.1 & 3.9 & 3.8 & 3.2 \\
\hline Musical instruments & 4 & $73 \%$ & 0.95 & $3 \%$ & $12 \%$ & $2 \%$ & $72 \%$ & 1.31 & 3.6 & 2.6 & 4.2 & 4.3 & 3.5 \\
\hline Natural elements and vegetation & 11 & $75 \%$ & 1.20 & $4 \%$ & $2 \%$ & $1 \%$ & $72 \%$ & 1.22 & 4.0 & 2.5 & 3.8 & 4.2 & 2.1 \\
\hline Outdoor activity and sport items & 19 & $63 \%$ & 1.50 & $0 \%$ & $4 \%$ & $2 \%$ & $68 \%$ & 1.40 & 3.9 & 2.1 & 4.3 & 4.2 & 3.2 \\
\hline Skin care and bathroom items & 30 & $55 \%$ & 1.74 & $2 \%$ & $5 \%$ & $2 \%$ & $78 \%$ & 0.98 & 4.3 & 2.1 & 4.2 & 4.0 & 3.7 \\
\hline Stationery and school supplies & 41 & $60 \%$ & 1.68 & $1 \%$ & $4 \%$ & $3 \%$ & $84 \%$ & 0.71 & 4.3 & 1.9 & 4.1 & 3.9 & 3.0 \\
\hline Weapons and items related to war & 0 & - & - & - & - & - & - & - & - & - & - & - & - \\
\hline Other & 40 & $70 \%$ & 1.24 & $2 \%$ & $2 \%$ & $1 \%$ & $49 \%$ & 1.89 & 4.0 & 2.2 & 4.1 & 3.9 & 2.9 \\
\hline
\end{tabular}

$\mathrm{NA}=$ name agreement; $\mathrm{DKO}=$ don't know object; DKN $=$ don't know name; $\mathrm{TOT}=$ tip of the tongue; $\mathrm{CA}=$ category agreement; Fam $=$ familiarity; $\mathrm{VC}=$ visual complexity; $\mathrm{OA}=$ object agreement; $\mathrm{VA}=$ viewpoint agreement; Man = manipulability

These correlations can be appreciated in Fig. 2, where the French and English ratings/agreements of most normative variables are plotted. This figure shows that the extent of the rating/agreement distribution (also reflected by the $S D \mathrm{~s}$ in Table 2) varied across norms. For instance, objects reached a wide variety of manipulability ratings, in contrast to visual complexity ratings, which were mainly limited to a score between 1 and 3. Looking at Fig. 2 also helps us detect "outlier" objects. Outliers were identified by computing the standardized residuals, with the French norms as the dependent variable and the English norms as the independent one. Standardized residuals beyond two standard deviations were
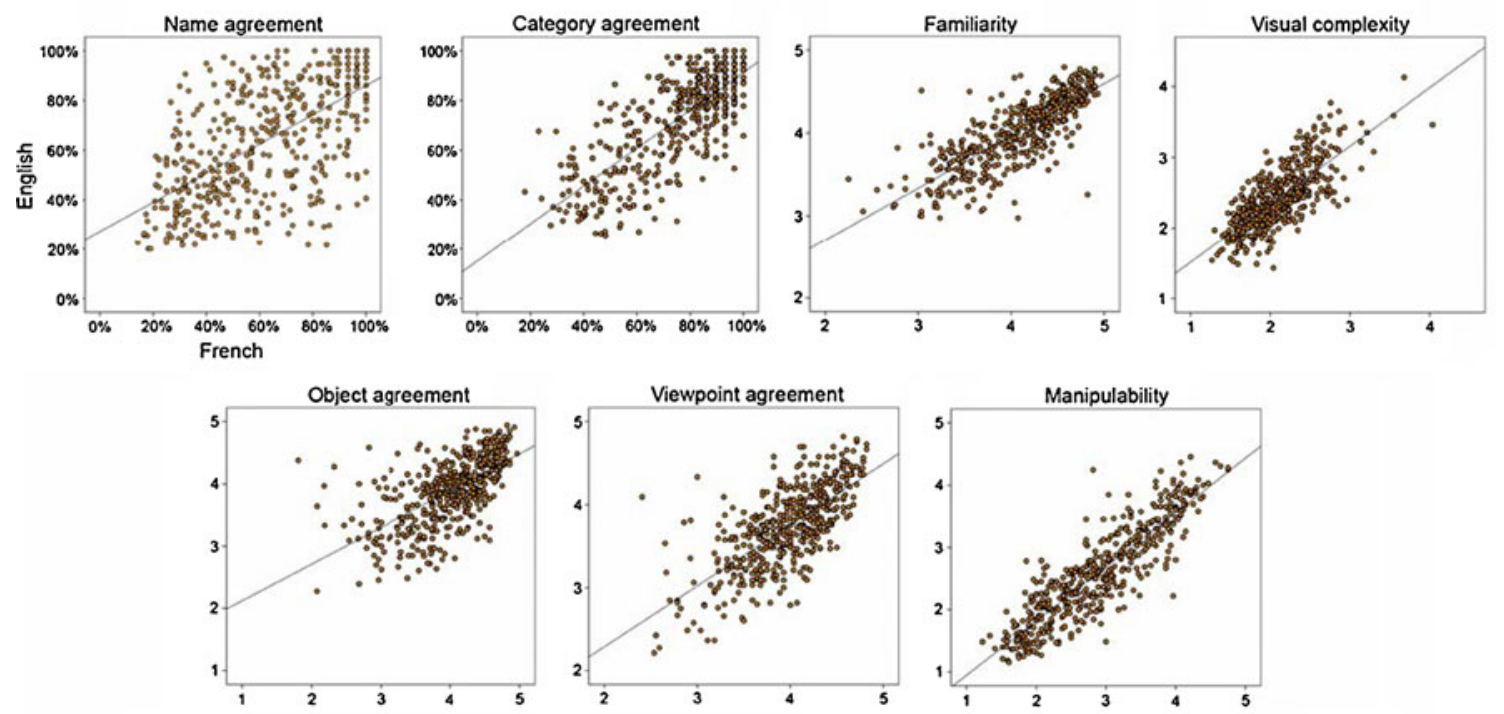

Fig. 2 Correlations between the ratings/agreement measures from English speakers ( $y$-axis) and French speakers ( $x$-axis). Each point corresponds to one photo from the BOSS 
classified as outliers. Outliers are identified in Appendix A by a superscript number; they were not removed from the correlational analyses.

\section{Discussion}

The present study proposes norms collected among French speakers living in the Montreal area and compares them with norms collected from English speakers also living in Montreal-thus, in a similar cultural environment. The first finding that arises is the high degree of similarity between the French and English norms. Mean name agreement was almost identical, and the $H$ values were not statistically different, implying that, overall, consensus on the name of an object and the use of alternative names were not different across languages. French and English speakers had the same extent of name agreement across objects (the $S D$ s were $24 \%$ and $23 \%$ in French and English, respectively), but Fig. 2 shows that name agreement did not necessarily apply to the same objects. In other words, there were approximately as many objects with, for example, $80 \%$ name agreement in French and English, but the objects reaching this rate of agreement were not systematically the same. However, the fact that the French and English name agreement norms did correlate indicates that objects with high name agreement in one language also had high name agreement in the other language.

The correlation in modal name agreement was .618, and thus well above all other correlations reported in studies that have compared name agreement for Snodgrass and Vanderwart (1980) pictures across different countries. For instance, the correlation between name agreement norms from French and American samples was .428 (Pompéia et al., 2003). Controlling for cultural setting between participant samples, as was done in the present study, likely increased the name agreement concordance between languages. Cultural differences could thus contribute to lowering the modal name agreement.

Like name agreement, category agreement and $H_{c a t}$ were not significantly different across linguistic groups, and the scores obtained in each language were highly correlated. We take this to imply that an object categorized, for example, as un meuble (furniture) in French was categorized, to approximately the same extent, as furniture in English. This norm was thus not really affected by the translation of the category list used during the normalization procedure. It is also of interest that all of the norms changed significantly as a function of category, even if the categories were not as contrasted (i.e., all of the stimuli were common objects) as those commonly used in the literature (e.g., animal vs. tool). Using only common objects as stimuli in an experiment is thus not a guarantee of good control for category effects.

Familiarity was also very comparable between languages. The mean rating was high in both languages, never reaching below 3.0. This was to be expected, given that the photos depict objects from everyday life. At .802, the correlation was also very high, meaning that objects familiar to the French speakers were also familiar to the English speakers. This result is consistent with the fact that the two groups share the same general environment, and are thus exposed daily to similar objects. The greater familiarity rating of the French Canadian participants as compared to the French (France) participants, described by Sirois et al. (2006), was thus likely due to a cultural effect. On the other hand, one must not neglect the fact that the present correlation was lower than the correlations between French and American individuals for the Snodgrass and Vanderwart (1980) pictures (Alario \& Ferrand, 1999). In our analyses, this result came as a surprise, since these two groups, in addition to having different languages, also have different cultures. It must be noted, however, that the Snodgrass and Vanderwart set offers simplified and prototypal representations of different concepts that are thought to be less subject to cultural influences than are the objects in the BOSS. We can reasonably assume that correlations between linguistic groups who are presented objects from the BOSS would be substantially reduced if these groups were from different countries. Moreover, as mentioned earlier, Alario and Ferrand removed objects that were uncommon in France from the normative set, thus increasing the chances of a higher correlation score.

Manipulability was not significantly different between linguistic groups, and its correlation of .886 was the highest of all of the norms. Like familiarity, manipulability can thus be considered unaffected by the mother tongue of the participants. This is not surprising, since familiarity and manipulability are known to be strongly interrelated. For instance, Magnié, Besson, Poncet, and Dolisi (2003) showed that manipulable objects are more familiar than unmanipulable objects. The homogeneous distribution of the manipulability ratings was, however, unexpected. Looking at Fig. 2, it can be seen that there are objects with almost all possible ratings, despite the fact that most objects depicted could have been held in one hand. It must, however, be remembered that, according to the instructions given to the participants, manipulability depended on the possibility of executing a specific action with the object, not simply on the possibility of holding it. For instance, a cauliflower can be handled but is not associated with a specific action.

Among the intergroup differences found in the present study, the difference in ratings of visual complexity (lower for French speakers than for English speakers) is challenging. This effect was not driven by some particular objects or by a category of objects (the Language $\times$ Category interaction in the ANOVA was not significant), but rather applies to all objects. As can be seen in Fig. 2, most ratings from the French speakers were between 1.5 and 2.5, whereas those of English speakers were between 2 and 3, and there were no 
more outliers with visual complexity than with the other normative variables. An effect of language cannot account for this intergroup difference, as rating visual complexity requires no specific semantic processing. What is particularly puzzling is the uniqueness of this difference relative to what has been found in the literature. Sirois et al. (2006), indeed, showed in their study comparing French and French Canadians norms that visual complexity was strongly affected by cultural settings. However, they also showed that cultural settings strongly affected familiarity. Thus, culture is unlikely to account for the visual complexity effect observed in our corpus, since there was no significant difference of familiarity. The visual complexity effect was also unlikely to be linked to a global response bias whereby English speakers used a higher end of the scale, as such bias would have also been observed with the other normative variables. One potential explanation could be that the instructions were understood differently, but this possibility cannot be tested empirically. It is important to note that the lower ratings from French speakers did not affect their correlation with the English speaker ratings. The most complex rated objects in French were thus also the most complex rated objects in English. This correlation was in the range of what has been reported so far in studies using line drawings (Pompéia et al., 2003). Therefore, despite a main effect of language, the French Canadian norms are still predictive of the English Canadian norms, and vice versa.

Viewpoint agreement was the only other normative variable that differed significantly between the French and English speakers. Unlike visual complexity, viewpoint agreement involved semantic processing of the word used to generate a mental image. This discrepancy of norms can thus easily be explained by the use of different words or by differences in their interpretation. However, word influence was not sufficient to cause group differences for object agreement. Thus, language appears to influence the position in which objects are imagined, but not their design. Moreover, object and viewpoint agreement among the French speakers correlated with agreement rates among the English speakers. To our knowledge, such correlations have not been computed between samples from different countries. We can, however, imagine that cultural differences would have a substantial impact on the correlations, because viewpoint and object agreement are very sensitive to the surrounding environment.

In conclusion, there is an overall good concordance between the norms collected with the BOSS from French and English native speakers living in a similar cultural setting. Some normative variables, such as familiarity and manipulability, are correlated to such an extent that one could reasonably argue for combining them. Despite the overall concordance, the present study has also exposed some effects that may pertain specifically to language differences; however, the magnitude of these effects is not as important as the ones reported by Sirois et al. (2006), which were mainly driven by cultural differences. The BOSS and the normative data set are available to researchers who wish to use them for developing their experimental stimuli and conditions.

Acknowledgment This study was supported by an operating grant from Natural Sciences and Engineering Research Council of Canada (grant \# 238617).

Author note We are grateful to Geneviève Charbonneau for having photographed and edited a number of the stimuli, to Thomas Howells and Jennifer Dell'Elce for manuscript editing, and to Christopher Taylor for calibration of the display system.

\section{References}

Alario, F.-X., \& Ferrand, L. (1999). A set of 400 pictures standardized for French: Norms for name agreement, image agreement, familiarity, visual complexity, image variability, and age of acquisition. Behavior Research Methods, Instruments, \& Computers, 31, 531552. doi:10.3758/BF03200732

Alario, F.-X., Ferrand, L., Laganaro, M., New, B., Frauenfelder, U. H., \& Segui, J. (2004). Predictors of picture naming speed. Behavior Research Methods, Instruments, \& Computers, 36, 140-155. doi:10.3758/BF03195559

Álvarez, B., \& Cuetos, F. (2007). Objective age of acquisition norms for a set of 328 words in Spanish. Behavior Research Methods, 39, 377-383. doi:10.3758/BF03193006

Barbarotto, R., Laiacona, M., Macchi, V., \& Capitani, E. (2002). Picture reality decision, semantic categories and gender: A new set of pictures, with norms and an experimental study. Neuropsychologia, 40, 1637-1653. doi:10.1016/S0028-3932(02)00029-5

Barry, C., Morrison, C. M., \& Ellis, A. W. (1997). Naming the Snodgrass and Vanderwart pictures: Effect of age of acquisition, frequency, and name agreement. Quarterly Journal of Experimental Psychology, 50A, 560-585. doi:10.1080/783663595

Bates, E., D’Amico, S., Jacobsen, T., Székely, A., Andonova, E., Devescovi, A., \& Tzeng, O. (2003). Timed picture naming in seven languages. Psychonomic Bulletin \& Review, 10, 344-380. doi:10.3758/BF03196494

Begleiter, H., Porjesz, B., \& Wang, W. (1995). Event-related brain potentials differentiate priming and recognition to familiar and unfamiliar faces. Electroencephalography and Clinical Neurophysiology, 94, 41-49. doi:10.1016/0013-4694(94)00240-L

Bonin, P., Chalard, M., Méot, A., \& Fayol, M. (2002). The determinants of spoken and written picture naming latencies. British Journal of Psychology, 93, 89-114. doi:10.1348/000712602162463

Bonin, P., Peereman, R., Malardier, N., Méot, A., \& Chalard, M. (2003). A new set of 299 pictures for psycholinguistic studies: French norms for name agreement, image agreement, conceptual familiarity, visual complexity, image variability, age of acquisition, and naming latencies. Behavior Research Methods, Instruments, \& Computers, 35, 158-167. doi:10.3758/BF03195507

Brainard, D. H. (1997). The psychophysics toolbox. Spatial Vision, 10, 433-436. doi:10.1163/156856897X00357

Brodeur, M. B., Dionne-Dostie, E., Montreuil, T., \& Lepage, M. (2010). The Bank of Standardized Stimuli (BOSS), a new set of 
480 normative photos of objects to be used as visual stimuli in cognitive research. PLOS ONE, 5, e10773. doi:10.1371/journal. pone. 0010773

Cuetos, F., \& Alija, M. (2003). Normative data and naming times for action pictures. Behavior Research Methods, Instruments, \& Computers, 35, 168-177. doi:10.3758/BF03195508

Cuetos, F., Ellis, A. W., \& Alvarez, B. (1999). Naming times for the Snodgrass and Vanderwart pictures in Spanish. Behavior Research Methods, Instruments, \& Computers, 31, 650-658. doi:10.3758/BF03200741

Cycowicz, Y. M., Friedman, D., Rothstein, M., \& Snodgrass, J. G. (1997). Picture naming by young children: Norms for name agreement, familiarity, and visual complexity. Journal of Experimental Child Psychology, 65, 171-237. doi:10.1006/ jecp.1996.2356

Dell'Acqua, R., Lotto, L., \& Job, R. (2000). Naming times and standardized norms for the Italian PD/DPSS set of 266 pictures: Direct comparisons with American, English, French, and Spanish published databases. Behavior Research Methods, Instruments, \& Computers, 32, 588-615. doi:10.3758/BF03200832

Ellis, A. W., \& Morrison, C. M. (1998). Real age-of-acquisition effects in lexical retrieval. Journal of Experimental Psychology: Learning, Memory, and Cognition, 24, 515-523. doi:10.1037/02787393.24 .2 .515

Greeno, J. G. (1994). Gibson's affordances. Psychological Review, 101, 336-342. doi:10.1037/0033-295X.101.2.336

Hannah, S. D., \& Brooks, L. R. (2009). Featuring familiarity: How a familiar feature instantiation influences categorization. Canadian Journal of Experimental Psychology, 63, 263-275. doi:10.1037/ a0017919

Magnié, M. N., Besson, M., Poncet, M., \& Dolisi, C. (2003). The Snodgrass and Vanderwart set revisited: Norms for object manipulability and for pictorial ambiguity of objects, chimeric objects, and nonobjects. Journal of Clinical and Experimental Neuropsychology, 25, 521-560. doi:10.1076/jcen.25.4.521.13873

Miranda, M. C., Pompéia, S., \& Bueno, O. F. (2004). A comparative study of norms for a 400 picture set between Brazilian and American children. Revista Brasileira de Psiquiatria, 26, 226-233.

Nishimoto, T., Miyawaki, K., Ueda, T., Une, Y., \& Takahashi, M. (2005). Japanese normative set of 359 pictures. Behavior Research Methods, 37, 398-416. doi:10.3758/BF03192709

Pind, J., Jónsdóttir, H., Gissurardóttir, H., \& Jónsson, F. (2000). Icelandic norms for the Snodgrass and Vanderwart (1980) pictures: Name and image agreement, familiarity, and age of acquisition. Scandinavian Journal of Psychology, 41, 41-48. doi:10.1111/ 1467-9450.00169
Pind, J., \& Tryggvadóttir, H. B. (2002). Determinants of picture naming times in Icelandic. Scandinavian Journal of Psychology, 43 221-226. doi:10.1111/1467-9450.00290

Pompéia, S., Miranda, M. C., \& Bueno, O. F. A. (2001). A set of 400 pictures standardised for Portuguese: Norms for name agreement, familiarity and visual complexity for children and adults. Arquivos de Neuro-Psiquiatria, 59, 330-337. doi:10.1590/S0004282X2001000300004

Pompéia, S., Miranda, M. C., \& Bueno, O. F. A. (2003). Brazilian standardised norms for a set of pictures are comparable with those obtained internationally. Arquivos de Neuro-Psiquiatria, 61, 916919. doi:10.1590/S0004-282X2003000600005

Rossion, B., \& Pourtois, G. (2004). Revisiting Snodgrass and Vanderwart's object pictorial set: The role of surface detail in basic-level object recognition. Perception, 33, 217-236. doi:10.1068/p5117

Schwitter, V., Boyer, B., Méot, A., Bonin, P., \& Laganaro, M. (2004). French normative data and naming times for action pictures. Behavior Research Methods, Instruments, \& Computers, 36, 564-576. doi:10.3758/BF03195603

Severens, E., Van Lommel, S., Ratinckx, E., \& Hartsuiker, R. J. (2005). Timed picture naming norms for 590 pictures in Dutch. Acta Psychologica, 119, 159-187. doi:10.1016/j.actpsy.2005.01.002

Sirois, M., Kremin, H., \& Cohen, H. (2006). Picture-naming norms for Canadian French: Name agreement, familiarity, visual complexity, and age of acquisition. Behavior Research Methods, 38, 300-306. doi:10.3758/BF03192781

Snodgrass, J. G., \& Vanderwart, M. (1980). A standardized set of 260 pictures: Norms for name agreement, image agreement, familiarity, and visual complexity. Journal of Experimental Psychology: Human Learning and Memory, 6, 174-215. doi:10.1037/02787393.6.2.174

van Elk, M., \& Blanke, O. (2011). Manipulable objects facilitate crossmodal integration in peripersonal space. PLoS One, 6, e24641.

Viggiano, M. P., Vannucci, M., \& Righi, S. (2004). A new standardized set of ecological pictures for experimental and clinical research on visual object processing. Cortex, 40, 491-509. doi:10.1016/ S0010-9452(08)70142-4

Weekes, B. S., Shu, H., Hao, M., Liu, Y., \& Tan, L. H. (2007). Predictors of timed picture naming in Chinese. Behavior Research Methods, 39, 335-342. doi:10.3758/BF03193165

Yoon, C., Feinberg, F., Luo, T., Hedden, T., Gutchess, A. H., Chen, H.-Y. M., . . Park, D. C. (2004). A cross-culturally standardized set of pictures for younger and older adults: American and Chinese norms for name agreement, concept agreement, and familiarity. Behavior Research Methods, Instruments, \& Computers, 36, 639-649. doi:10.3758/BF03206545 